\title{
Spur Reduction in Wideband PLLs by Random Positioning of Charge Pump Current Pulses
}

\author{
Chembiyan Thambidurai \& Nagendra Krishnapura \\ Department of Electrical Engineering, Indian Institute of Technology, Madras, Chennai 600 036, India
}

\begin{abstract}
Charge pump PLL is prone to reference spurs due to non-idealities like feedthrough, charge pump current mismatch and loop filter leakage. To resolve the problem of reference spurs, a randomization technique that converts the spurs to wideband noise is proposed. The added wideband noise has insignificant contribution to the output phase noise inside the PLL bandwidth. Analytical expressions are derived to study the effect of the randomization on the output phase noise. A $1 \mathrm{GHz}$ output frequency PLL with a bandwidth of $1 \mathrm{MHz}$ and a 20 $\mathrm{MHz}$ reference frequency is simulated to test the idea. From the simulated results we could see a spur reduction of $20 \mathrm{~dB}$ in the nominal case. The settling time of the simulated PLL measured for a $25.2 \mathrm{ppm}$ settling error is $4.5 \mu \mathrm{s}$.
\end{abstract}

\section{INTRODUCTION}

In the steady state of a charge pump PLL when the divide and reference edges align, the charge pump current should ideally be zero. But in reality due to the circuit non-idealities like charge pump current mismatch, loop filter leakage, feedthrough of the charge pump switches, a non zero current $\left(i_{\text {cp }}(t)\right)$ with zero average value is injected into the loop filter as shown in Fig. 1. The charge pump current injected into the loop filter every reference cycle $\left(T_{r}\right)$ is a pair of impulses (narrow triangular pulses) spaced reset delay $\left(\mathrm{T}_{\mathrm{rst}}\right)$ apart in case of the feedthrough $\left(\mathrm{I}_{\mathrm{ft}}\right)$ of charge pump switches and a pulse in presence of charge pump current mismatch $\left(\mathrm{I}_{\text {mis }}\right)$ or loop filter leakage $\left(\mathrm{I}_{\text {leak }}\right)$ as shown in the Fig. 1(a), 1(b) and 1(c) respectively. This non-zero current generates a periodic disturbance on the control voltage and manifests itself as a reference spur at the PLL output.

The magnitude of the spur at a frequency offset $f_{r}$ from the carrier at the PLL output can be expressed in $\mathrm{dBc}$ as [1]

$$
S_{\phi}\left(f_{r}\right)=20 \log \left(\frac{I_{c p}\left(f_{r}\right)\left|Z\left(f_{r}\right)\right| K_{v c o}}{2 f_{r}}\right)
$$

Where $I_{c p}\left(f_{r}\right)$ is the ac magnitude of the charge pump current at a frequency $f_{r}$. Equation (1) implies that a low spur level at the output is achieved by having a low VCO gain $\left(K_{v c o}\right)$ or a low loop filter impedance $\left(Z\left(f_{r}\right)\right)$. But reducing these two parameters reduces the bandwidth of the PLL, leading to longer settling time. And there appears a trade-off between settling time and tolerable reference spur level. Several techniques have been proposed to mitigate this problem. [2] addresses the issue based on the technique of delay-sampling the control voltage. But this technique is not effective in the presence of loop filter capacitor leakage. [3] minimizes the charge pump mismatch thereby reducing the spur, at the cost of increased settling time. [4] uses distributed charge pump

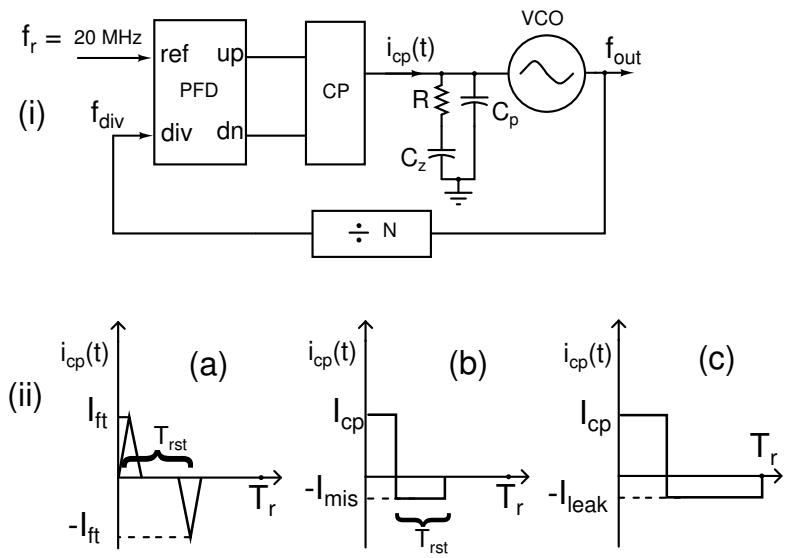

Fig. 1. (i) The standard charge pump PLL. (ii) Current injected into the loop filter due to (a) Charge pump feedthrough (b) Charge pump mismatch (c) Loop filter leakage

and PFD with pulse position randomization to reduce the spur. Using distributed PFD and charge pumps can cause the total size of the charge pump switches to be larger than the single one, increasing the net feedthrough error besides an increase in implementation complexity.

The present work proposes a method of achieving low spur levels without compromising on settling time by randomizing the position of charge pump current pulses. This technique will be effective even in the presence of loop filter leakage and uses a single charge pump and PFD. Section II explores the theoretical basis for the idea. Section III describes the implementation. Section IV shows the simulation results. Section V delves into the effects of non-idealities in the implementation on the performance of the PLL. Section VI presents the conclusions drawn from the work.

\section{Random Pulse Positioning}

As discussed in the Section I, the periodicity in the steady state charge pump current is the cause of the spurs at the output. The central idea of this work is to break the periodicity by randomly placing the current pulses within the reference period. In theory this randomizing effect redistributes the energy of the current pulses concentrated at the harmonics of reference frequency to all the frequencies making it appear as a wideband noise. And the resulting "redistributed noise" is further filtered by the loop-filter and VCO as given in equation (1). Ideally this approach can significantly reduce the magnitude of the spur. But in reality the phase shifts of 
$x(t)$

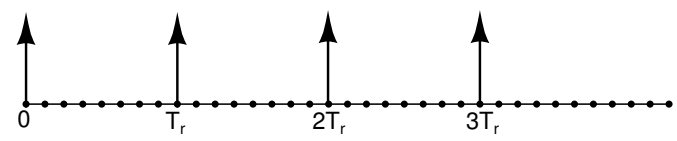

$r(\mathrm{t})$

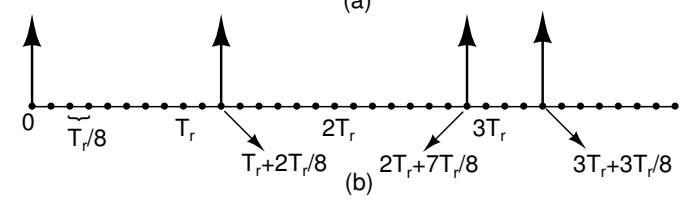

Fig. 2. (a) Periodic impulse train. (b) Random impulse position modulation.
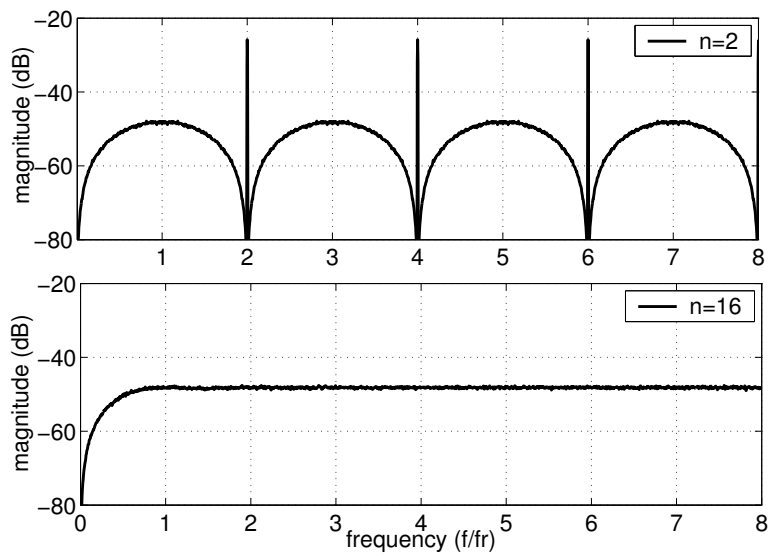

Fig. 3. The spectrum of random impulse positioning for $n=2 \& n=16$ (PSD computed with a resolution binwidth of $f_{r} / 256$ )

the current pulses cannot be continuous but a multiple of a fixed number. The effects of this quantization in phase shift is discussed below.

The reference period $\left(T_{r}\right)$ is divided into small and equal time intervals of value $T_{r} / n$ and the $k^{\text {th }}$ current pulse will now appear at $k T_{r}+a_{k} T_{r} / n$ instead at $k T_{r} . a_{k}$ is a uniform random number that can take integer values in the interval $[0, n-1]$. The periodic current pulses can be modelled as a periodic impulse train. The impulse train and the associated random signal are shown in the Fig. 2 for $n=8$. The periodic signal and the random signal can be expressed as

$$
\begin{gathered}
x(t)=\sum_{k=-\infty}^{\infty} \delta\left(t-k T_{r}\right) \\
r(t)=\sum_{k=-\infty}^{\infty} \delta\left(t-k T_{r}-\frac{a_{k} T_{r}}{n}\right)
\end{gathered}
$$

where $a_{k} \in[0, n-1]$. The power spectrum of the signal $x(t)$ is that of an impulse train and is given by

$$
S_{x}(f)=\frac{1}{T_{r}^{2}} \sum_{k=-\infty}^{\infty} \delta\left(f-\frac{k}{T_{r}}\right)
$$

The spectrum of the random signal $r(t)$ can be represented as

$$
S_{r}(f)=S_{r d}(f)+\frac{1}{T_{r}^{2}} \sum_{k=-\infty}^{\infty} \delta\left(f-k \frac{n}{T_{r}}\right)
$$

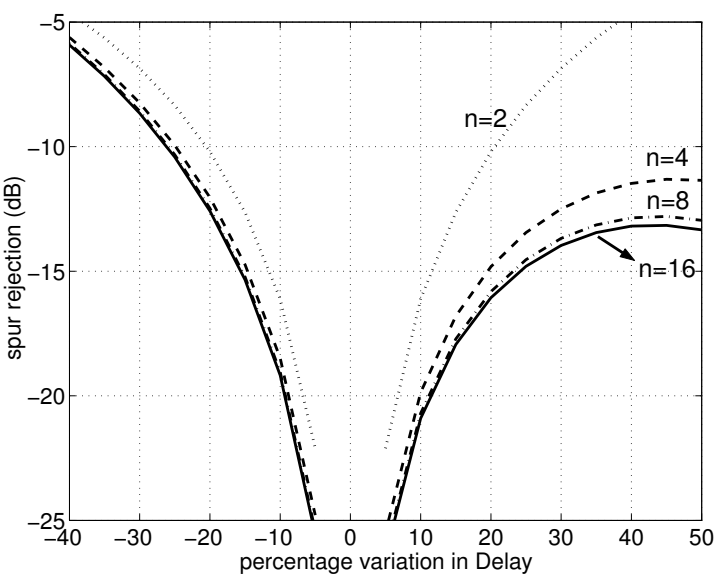

Fig. 4. Spur rejection vs percentage delay variation for different values of $n$

where $S_{r d}(f)$ is the "redistributed noise" and

$$
S_{r d}(f)=\frac{1}{n T_{r}}\left[(n-1)-\frac{2}{n} \sum_{k=1}^{n-1}(n-k) \cos \left(\frac{2 \pi k f T_{r}}{n}\right)\right]
$$

For a simple case $n=2$,

$$
S_{r d}(f)=\frac{1}{T_{r}} \sin ^{2}\left(\frac{\pi f T_{r}}{2}\right)
$$

(The proof of equations (5) and (6) for $n=2$ is derived in Appendix I). From equations (4) and (5), we see that the spectrum before randomization contains harmonics at $f_{r}$ and the spectrum after randomization contains harmonics at $n f_{r}$ and the energy present in the harmonics $f_{r}$ to $(n-1) f_{r}$ is spread in the band 0 to $n f_{r}$. The simulated spectrum of the randomized signals is shown in Fig. 3 for $n=2$ and $n=16$. For any arbitrary pulse shape of the charge pump current, the spectrum after randomization is obtained by multiplying the spectrum shown in Fig. 3 with the spectrum of the charge pump current pulse. Then using equation (1) we can obtain the phase noise contribution after randomization at the PLL output. We see from equation (7) and also from Fig. 3 that the "redistributed noise" after randomization is not white but rather shaped in frequency (by a $\sin ^{2}$ function for $n=2$ ) and possesses insignificant energy at frequencies close to DC. Hence the randomization doesn't affect the close-in phase noise (long-term jitter) of the PLL.

In the preceding analysis, the delay value is assumed to be fixed. But in reality the delays are prone to process variations as they are implemented using inverters. If the implemented delay is not equal to $T_{r} / n$, then spurs appear in the output as explained in Section V. Fig. 4 shows the spur rejection vs percentage variations in the delay from its ideal value. From Fig. 4 it can be seen that for delay variations greater than than the ideal value, there is an improvement of $5 \mathrm{~dB}$ for $n=4$, and $6.5 \mathrm{~dB}$ for $n=8$ when compared to $n=2$ in the spur rejection, for $30 \%$ variation in the delay. As $n$ increases, we get better rejection even for larger variations in the delay. But the increase becomes marginal for $n$ greater than 8 . We chose $n=16$ for our simulations for less sensitivity to delay variations. 


\section{IMPLEMENTATION}

As discussed in Section II, the position of the charge pump current pulses is varied randomly within a reference cycle to reduce the spur. This is nothing but shifting the charge pump current position by the multiples of fixed delay. This can be easily achieved by delaying the 'up' and 'dn' pulses of the PFD and choosing one pair (up/dn) of the delayed pulses randomly. We use a 15-cell delay chain with the delay value of each cell equal to $T_{r} / 16$. And one of the 16 possible

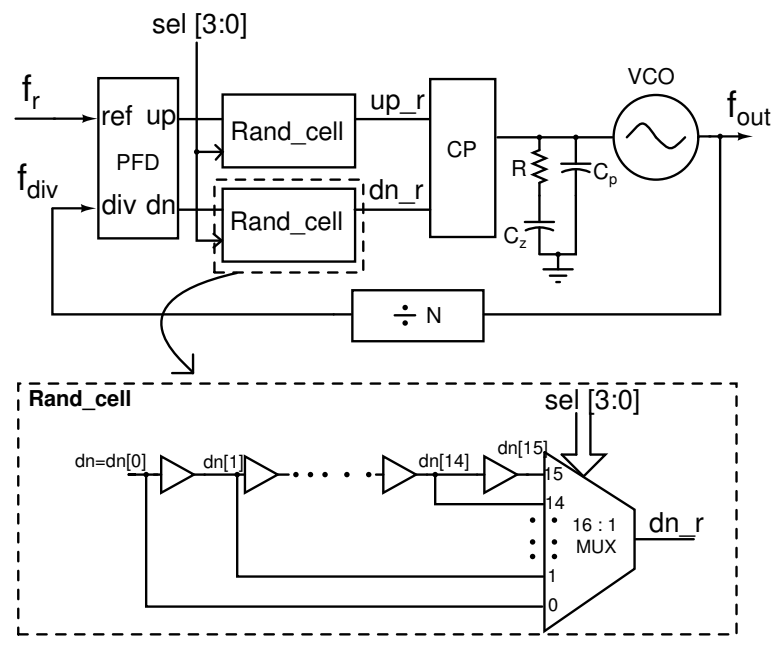

Fig. 5. Modified PLL architecture

versions of the 'up' and 'dn' signals is chosen randomly per reference cycle using a 16:1 multiplexer based on a random 4bit control signal (sel[3:0]) generated by a $\mathrm{PRBS}^{1}$ generator. The modified architecture of the PLL with the randomization technique included is shown at a system level in Fig. 5.

\section{Simulation Results}

To validate the proposed idea, a PLL was simulated with $1 \mathrm{MHz}$ bandwidth running at an output frequency of $1 \mathrm{GHz}$ and a reference frequency of $20 \mathrm{MHz}$. The charge pump current $\left(I_{c p}\right)$ is $50 \mu A$, the loop-filter parameters are $\mathrm{R}=21.7 \mathrm{k} \Omega, \mathrm{C}_{\mathrm{z}}=37.25 \mathrm{pF}, \mathrm{C}_{\mathrm{p}}=1.99 \mathrm{pF}$. The $\mathrm{VCO}$ gain, $K_{v c o}=200 \mathrm{MHz} / \mathrm{V}$. The nominal divide value is $N=50$. The PFD, CP and loop filter are at transistor level and the remaining blocks (VCO, divider and the digital logic for randomization) are modelled behaviorally. The VCO, divider and the randomization logic are assumed to be ideal (noiseless) and the source of noise is only deterministic, contributed by the charge pump. To model the effect of charge pump mismatch, a constant current source of value $0.1 I_{c p}$ is connected in parallel to the sourcing current. The feedthrough is inherent with the

\footnotetext{
${ }^{1}$ The randomizing sequence should posses a uniform distribution for perfect spur elimination. A single PRBS generator with 4 taps taken from different points of the LFSR (Linear Feedback Shift Register) of length greater than 7 can guarantee a near uniform distribution but not a white spectrum (which leads to a slight increase in the in-band noise). Four PRBS generators with different state transitions can be used to generate the 4-bit random data with white spectrum. A larger PRBS length lowers the in-band noise floor. The length of the PRBS is determined by the noise floor that can be tolerated by the system. (Details not given due to space constraints).
}

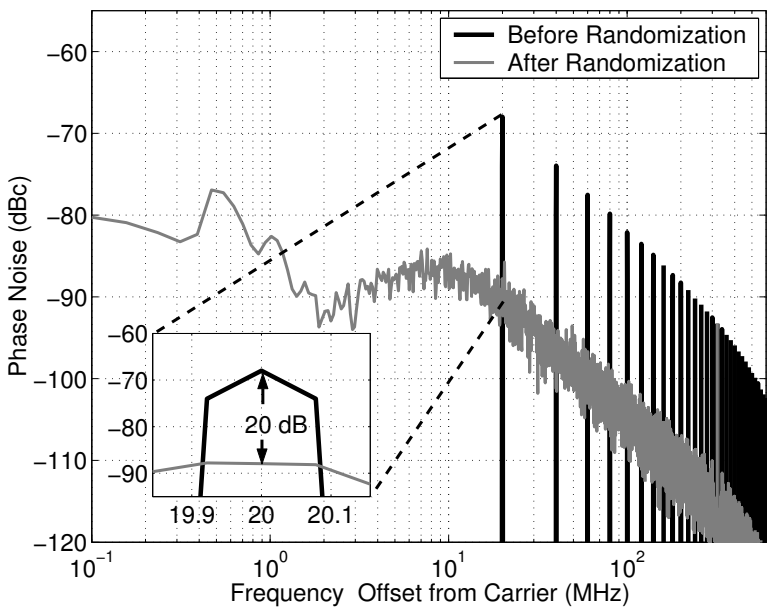

Fig. 6. Phase noise of the PLL output comparing the the performance with and without randomization. (The resolution bandwidth used for PSD computation is $78.125 \mathrm{kHz}$ )

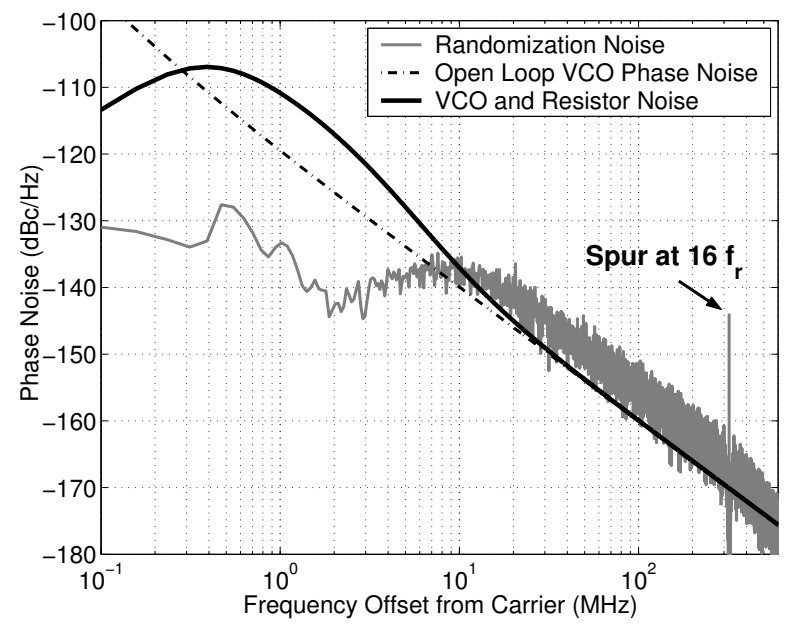

Fig. 7. Phase noise at the PLL output due to resistor and VCO compared with the "spread noise" due to randomization

circuit of the implemented charge pump. The simulated phase noise with and without randomization is shown in Fig. 6 in $\mathrm{dBc}$. The simulated rejection of the spur was $20 \mathrm{~dB}$. It corroborates our claim of dealing with all the non-idealities of the PLL that produce reference spur.

To study the effect of the technique on the output phase noise at high frequencies we compare the phase noise contribution of the VCO and loop filter resistor to the randomization noise. Fig. 7 shows the phase noise due to the randomization overlaid with the resistor and VCO noise at the PLL output. (The phase noise model of the open loop VCO assumes a phase noise specification of $120 \mathrm{dBc} / \mathrm{Hz}$ at $1 \mathrm{MHz}$ offset and a $1 / f^{3}$ corner of $200 \mathrm{kHz}$ as shown in Fig. 7). The phase noise contributed to the PLL output by VCO and resistor is dominant near the bandwidth of the PLL and also at high frequencies. The plot shows that the noise added by the randomization is at least 20 $\mathrm{dB}$ lower than these two contributions at frequencies close to the bandwidth of the PLL and it becomes dominant only at frequencies greater than $10 \mathrm{MHz}$. 


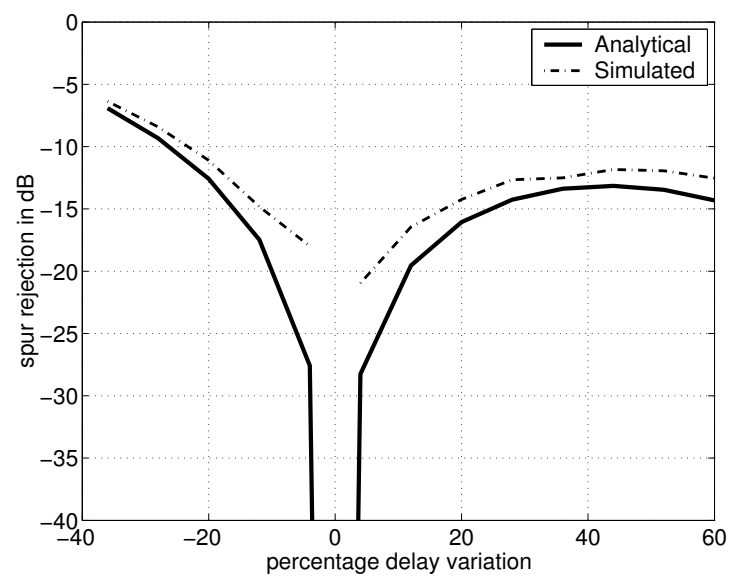

Fig. 8. Spur rejection vs delay variation

\section{EFFECT OF DELAY VARIATION}

The delay cells are implemented using inverters and are thus prone to process variations. To understand the effect of the delay variations, the randomization over a large number of reference cycles can be seen as a moving average filter (since we choose one of the possible $\mathrm{n}$ delayed versions of the charge pump current pulse randomly). Let $i_{c p}(t)$ be the charge pump current waveform and the random number selecting the shifted waveforms be uniformly distributed. Then, on an average the current after randomization $\left(i_{r}(t)\right)$ can be written as

$$
i_{r}(t)=\frac{1}{n}\left[i_{c p}(t)+i_{c p}\left(t-T_{d}\right)+\ldots . .+i_{c p}\left(t-\frac{(n-1) T_{d}}{n}\right)\right]
$$

And the resulting spectrum of $i_{r}(t)$ can be expressed as

$$
\left|I_{r}(f)\right|=\frac{\sin \left(n \pi f T_{d}\right)}{\sin \left(\pi f T_{d}\right)}\left|I_{c p}(f)\right|
$$

From equation (9) we can see that the filtering action introduces zeroes at frequencies $f_{z}=k / n T_{d}$ where $\mathrm{k}$ is an integer and $k \neq 0, n, 2 n \ldots .$, . When $T_{d}=T_{r} / n$, the zeroes occur exactly at the multiples of $f_{r}$ and the spurs are completely eliminated. But when $T_{d} \neq T_{r} / n$, the zeroes of the filter do not occur at multiples of $f_{r}$ and this leads to incomplete elimination of spurs. Fig. 8 shows the variation of the spur rejection with the change in the delay from its ideal value. From simulations we could see at least a rejection of $11.5 \mathrm{~dB}$ for $20 \%$ variation in the delay.

\section{CONCLUSION}

A technique to reduce the reference spur by randomlyvarying the position of the charge pump current pulse within a reference cycle has been proposed. The spectrum thus obtained after the randomization is derived and its effect on the output phase noise is discussed. The implementation of the technique is discussed at block level. A quantitative analysis is presented on the effects of delay variations in the charge pump pulse positions. For a given bandwidth this technique results in 20 $\mathrm{dB}$ reduction in the nominal case and least $11.5 \mathrm{~dB}$ reduction for $20 \%$ variation in the delays of the logic. Alternatively for a given spur level, using this technique, the bandwidth can be made larger resulting in faster settling.

\section{APPENDIX I}

From Section II we can write the expression for the random impulse position modulated waveform as

$$
r(t)=\sum_{k=-\infty}^{\infty} \delta\left(t-k T_{r}-b_{k} \frac{T_{r}}{2}\right)
$$

For $n=2, b_{k}$ is a random variable with $P\left(b_{k}=1\right)=P\left(b_{k}=\right.$ $0)=0.5$, and $b_{k}, b_{k+1}$ are independent for all $k$.

To solve this problem in a simpler way we converted the randomness in the position of the impulse to the randomness in amplitude of the impulse. Thus equation (10) can be rewritten as

$$
r(t)=\sum_{k=-\infty}^{\infty} a_{k} \delta\left(t-\frac{k T_{r}}{2}\right)
$$

This is a pulse amplitude modulated (PAM) signal with the pulse being an impulse and the symbol period equal to $0.5 T_{r}$. $a_{k}$ is a random variable with $P\left(a_{k}=1\right)=P\left(a_{k}=0\right)=0.5$. But the impulse cannot occur at $2 k$ or $2 k+1$ simultaneously. This condition can be translated into a constraint that $P\left(a_{2 k}=1\right)=$ 0.5 and $P\left(a_{2 k+1}=1 \mid a_{2 k}=1\right)=0$. The same holds true for $a_{2 k}=0$ vice versa. From [6] we know the spectrum for a PAM signal of period $T_{r} / 2$ is given by

$$
S_{r}(f)=\frac{2}{T_{r}}|P(f)|^{2} \sum_{m=-\infty}^{\infty} \hat{R}_{r}(m) e^{-\pi f T_{r}}
$$

$|P(f)|=1$ for an impulse (The illustrative pulse shapes are given in Fig. 1 of Section I). And the autocorrelation function $\hat{R}_{r}(m)$ is given by

$$
\hat{R}_{r}(m)=E[r(k) r(k+m)]
$$

Using the constraints mentioned above, we get the autocorrelation function as $\hat{R}_{r}(0)=0.5, \hat{R}_{r}( \pm 1)=0.125$ and $\hat{R}_{r}(m)=0.25$ for $m \neq \pm 1,0$ Substituting this in equation (12) and after some manipulations we obtain

$$
\left.S_{r}(f)=\frac{1}{T_{r}^{2}} \sum_{k} \delta\left(f-k \frac{2}{T_{r}}\right)+\frac{1}{T_{r}} \sin ^{2}\left(\frac{\pi f T_{r}}{2}\right)\right]
$$

Proceeding in a similar manner, one can show that for any $n$, the spectrum $S_{r}(f)$ can be expressed as given in equations (5) and (6) of Section II.

\section{REFERENCES}

[1] Cicero.S.Vaucher, "An adaptive PLL tuning system architecture combining high spectral purity and fast settling time." IEEE Journal of Solid State Circuits, pp. 2131-2137, vol. 35, issue 4, April. 2000.

[2] Che-Fu Liang et al., "Spur suppression techniques for frequency synthesizers," IEEE Transactions on Circuits and Systems-II:Express Briefs, pp. 653-657, vol. 54, issue 8, Aug. 2007.

[3] Che-Fu Liang et al., "A digital calibration technique for charge pumps in phase-locked systems," IEEE Journal of Solid State Circuits, pp. 390398, vol. 38, issue 2, Feb. 2008.

[4] T. C. Lee, W. L. Lee, "A spur suppression technique for phase Locked frequency synthesizers.", International Solid State Circuits Conference, ISSCC 2006, pp.592-593.

[5] Cameron T. Charles, David J Allstot, "A Calibrated Phase/Frequency Detector for Reference Spur Reduction in Charge-Pump PLLs," IEEE Transactions on Circuits and Systems-II:Express Briefs, vol. 53, issue 9, Sep. 2006.

[6] B. P. Lathi, Modern Digital and Analog Communication Systems., Third edition, New York:Oxford University Press., 1998. 